\title{
Qualitative and Quantitative Analysis of Five Bioactive Flavonoids in Salix bordensis Turcz. by HPLC-DAD and HPLC-ESI-MS
}

\author{
Lei Zhao ${ }^{*}$, Lina Liu' ${ }^{2}$, Jintian $\mathrm{Li}^{3}$ \\ ${ }^{1}$ Key Laboratory of Chemistry and Quality for Traditional Chinese Medicines of the College of Gansu Province, \\ Gansu College of Traditional Chinese Medicine, Lanzhou, China \\ ${ }^{2}$ The Infectious Disease Hospital of Gansu Province, Lanzhou, China \\ ${ }^{3}$ Key Laboratory of Dunhuang Medicine and Transformation of Ministry of Education, Gansu College of \\ Traditional Chinese Medicine, Lanzhou, China \\ Email: ${ }^{\text {luckleizhao@126.cn }}$
}

Received 4 July 2014; revised 22 August 2014; accepted 9 September 2014

Copyright (C) 2014 by authors and Scientific Research Publishing Inc.

This work is licensed under the Creative Commons Attribution International License (CC BY).

http://creativecommons.org/licenses/by/4.0/

(c) (i) Open Access

\section{Abstract}

The qualitative characterization and quantitative analysis of five bioactive flavonoids in Salix bordensis Turcz. were achieved via reversed-phase high-performance liquid chromatography coupled with diode array detection and tandem mass spectrometry, by using an Agilent ZORBAX SB-C ${ }_{18}$ HPLC column with a gradient elution of $0.3 \%(\mathrm{v} / \mathrm{v})$ formic acid in water and methanol as the mobile phase. The compounds in the mixture were clearly identified by comparing their HPLC-DAD ultraviolet spectra, retention times, and MS data with those of corresponding reference compounds. All calibration curves showed good linearity $\left(r^{2}>0.9998\right)$ within the test ranges. The LOD, LOQ, specificity, precision, and accuracy for the method were validated. The results demonstrated that this analytical approach is ideal for the determination of bioactive compounds, such as flavonoids, and that it constructed a basis for the comprehensive evaluation of the quality of Salix bordensis Turcz.

\section{Keywords}

Flavonoids, Salix Bordensis Turcz., Qualitative and Qualitative Analysis, High-Performance Liquid Chromatography-Diode Array Detection, High-Performance Liquid-Chromatography-Electrospray Tandem Mass Spectrometry

${ }^{*}$ Corresponding authors. 


\section{Introduction}

Flavonoid is a widespread family of plant secondary metabolites, and it is always conjugated to glycosides. Flavonoids comprise several subclasses, which include flavones, isoflavones, flavonols, flavanones, and so on. These classes are grouped based on the degree of unsaturation and oxidation of the three-carbon segment of C6-C3-C6 flavone skeleton [1] [2]. Many flavonoids have biological activities. For example, kaempferol shows an anti-inflammatory effect on activated macrophages [3], has chemopreventive properties in brain tumors [4], and has an suppression effect on cell proliferation in human gut cancer lines [5] [6]. Similarly, quercetin also has various pharmacological actions, including scavenging of free radicals [7]-[9], modulation of epoxyeicosanoic acid synthesis, prevention of platelet aggregation [8], and vasodilating effects [10] [11].

Our main interest in this study is in the flavonoids found in Salix bordensis Turcz., a woody plant of the Salix (Salicaceae) family, which is a variation of Salix microstachya Turcz. It is distributed in the northwest (Gansu, Qinghai and Ningxia province, China) and northeast (Heilongjiang, Jilin and Liaoning province, China) of China and used in the production of fiber and energy. It has a number of desirable characteristics, which include a fast growth rate, short rotation cycle, ease of clonal propagation, high drought and disease resistance, and tight coupling between physiological traits and biomass productivity. Its root and fibrous roots can clear away heat, along with gas, and it has been used in folk medicine for the treatment of gastropyretie toothache and acute lumbar muscle sprain [12]. Previous studies have focused on the drought resistance, wind prevention, and sand fixation of Salix bordensis Turcz. [13]-[16]. Except for studies on the isolation and identification of ten compounds, including seven flavonoids by Luo et al. [17] and Yang et al. [18], systematic investigations on the phytochemistry of the components of Salix bordensis Turcz. have not been carried out properly, and no reports on the simultaneous quantitation of flavonoids are currently available. The primary focus of this study is to establish a platform for the analysis of identified flavonoids from Salix bordensis Turcz. in Gansu province, China. The objective is to provide preliminary data for a comprehensive HPLC-MS analysis of flavonoids in Salix bordensis Turcz. and to lay a scientific and technical basis for the utilization and development of the plant resources of the Salix (Salicaceae) family.

\section{Experimental}

\subsection{Chemicals and Materials}

High-performance liquid chromatography (HPLC)-grade acetonitrile and methanol (E. Merck, Darmstadt, Germany) were used for the HPLC analysis. Deionized water was purified using a Milli-Q system (Millipore, Bedford, MA, USA). All other chemicals were of analytical grade and were purchased from Tianjin Reagent Company (Tianjin, China). The five reference substances, namely, quercetin-3-O- $\beta$-D-glucoside (f1), quercetin (f2), kaempferol (f3), rhamnetin (f4), and kaempferol-7,4'-dimethyl (f5), were isolated from the Salix bordensis Turcz. in Lanzhou University. The structures of the five flavonoids are shown in Table 1 . The purity of all compounds exceeded 98\% (determined via HPLC). The structures of the compounds were confirmed by data from mass spectrometry (MS), proton nuclear magnetic resonance spectroscopy $\left({ }^{1} \mathrm{H}-\mathrm{NMR}\right)$, and carbon nuclear magnetic resonance spectroscopy $\left({ }^{13} \mathrm{C}-\mathrm{NMR}\right)$ experiments compared with those reported in literature [17].

The samples of Salix bordensis Turcz. were collected from Zhangye City, Gansu Province and were dried before extraction at $40^{\circ} \mathrm{C}$ until a constant weight was obtained in the dark. The botanical origins of the material were identified by Professor Zhigang Ma at Lanzhou University (Lanzhou, China). The voucher specimens were deposited at the Key Laboratory of Chemistry and Quality for Traditional Chinese Medicines of the College of Gansu Province, Gansu College of Traditional Chinese Medicine, Lanzhou, China.

\subsection{High-Performance Liquid Chromatography-Diode Array Detection Analysis}

High-performance liquid chromatography-diode array detection (HPLC DAD) analysis was performed using an Agilent 1100 series HPLC instrument (Agilent, Waldbronn, Germany) equipped with a G1312A binary pump, a G1315B diode array detector, a G1329A autosampler, and a G1316A column compartment. Data acquisition was done using a copyrighted Agilent Technologies software.

Separation was performed using a ZORBAX SB- $\mathrm{C}_{18}$ column $(5 \mu \mathrm{m}, 250 \mathrm{~mm} \times 4.6 \mathrm{~mm}$, i.d., Agilent, USA). The temperature of the column was set at $30^{\circ} \mathrm{C}$. A gradient system with $0.3 \%(\mathrm{v} / \mathrm{v})$ formic acid in water (A) and 
methanol (B) was used, as shown in Table 2. The mobile phase flow rate for the separation was $1 \mathrm{~mL} \cdot \mathrm{min}^{-1}$. The injection volume was $20 \mu \mathrm{L}$. Chromatograms were recorded at an absorbance of $360 \mathrm{~nm}$. In this work, all calculations for quantitative analysis were performed with linear regression external standardization by measuring the peak areas. The samples were filtered through a $0.45 \mu \mathrm{m}$ HPLC filter before injection.

\subsection{MS Condition}

High-performance liquid chromatography-electrospray ionization tandem mass spectrometry (HPLC-ESI/MS) experiments were performed using the Agilent 1100 HPLC system described above, combined with a Bruker Esquire 3000 Plus ion trap mass spectrometer (Bruker Franzen Analytik GmbH, Bremen, Germany) equipped with an electrospray ionization (ESI) interface. Instrument control and data acquisition were performed using the Esquire 5.0 software. The ion source temperature was $260^{\circ} \mathrm{C}$, and the ESI needle voltage was always set at -4.0 $\mathrm{kV}$. Nitrogen was used for drying, and the nebulizer gas had a flow rate of $10 \mathrm{~L} / \mathrm{min}$ and a back-pressure of 30 psi. Helium was introduced into the trap to function as the collision gas with an estimated pressure of $6 \times 10^{-6}$ mbar to improve trapping efficiency. For HPLC-ESI/MS analysis, the flow was subjected to a split of 1:5 before being introduced into the ion source. A short delay of approximately 0.2 min occurred between the DAD and MS systems as a consequence of the connecting tubing between HPLC and MS.

Table 1. Chemical structure of the five quantified compounds.<smiles>[R20]c1cc(O)c2c(=O)c([R2])c(-c3ccc([R6])c([R3])c3)oc2c1</smiles>

\begin{tabular}{ccccccccc}
\hline & Compounds & $\mathrm{R} 1$ & $\mathrm{R} 2$ & $\mathrm{R} 3$ & $\mathrm{R} 4$ & $\mathrm{RT}(\mathrm{min})$ & Quasimole cularion (m/z) & M.W. \\
\hline f1 & quercetin-3-O- $\beta$-D-glucopyranuronide & $\mathrm{Oglc}$ & $\mathrm{OH}$ & $\mathrm{OH}$ & $\mathrm{OH}$ & 18.868 & $479[\mathrm{M}+1]^{+}$ & 478 \\
f2 & quercetin & $\mathrm{OH}$ & $\mathrm{OH}$ & $\mathrm{OH}$ & $\mathrm{OH}$ & 31.991 & $303[\mathrm{M}+1]^{+}$ & 302 \\
f3 & kaempferol & $\mathrm{OH}$ & $\mathrm{OH}$ & $\mathrm{H}$ & $\mathrm{OH}$ & 37.043 & $287[\mathrm{M}+1]^{+}$ & 286 \\
f4 & rhamnetin & $\mathrm{OH}$ & $\mathrm{OCH}_{3}$ & $\mathrm{OH}$ & $\mathrm{OH}$ & 41.139 & $317[\mathrm{M}+1]^{+}$ & 316 \\
f5 & kaempferol-7,4'-dimethyl & $\mathrm{OH}$ & $\mathrm{OCH}_{3}$ & $\mathrm{H}$ & $\mathrm{OCH}_{3}$ & 48.047 & $315[\mathrm{M}+1]^{+}$ & 314 \\
\hline
\end{tabular}

*glc: glucopyranuronide; M.W., molecular weight.

Table 2. Solvent gradient program of HPLC analysis.

\begin{tabular}{ccc} 
Time (min) & A (\%) & B (\%) \\
\hline 0 & 68 & 32 \\
20 & 60 & 40 \\
35 & 40 & 60 \\
50 & 0 & 100 \\
60 & 0 & 100 \\
\hline
\end{tabular}




\subsection{Preparation of Standard and Sample Solutions}

Appropriate amounts of standards were accurately weighed and dissolved in $80 \%(\mathrm{v} / \mathrm{v})$ methanol-water to prepare the stock solutions for five reference standards. The solutions for the chromatographic runs were diluted from the stock solutions with $80 \%(\mathrm{v} / \mathrm{v})$ methanol-water.

The Salix bordensis Turcz. sample was mechanically ground. An accurately weighed sample was ultrasonically extracted for $40 \mathrm{~min}$ with ten times $80 \%(\mathrm{~m} / \mathrm{v} \mathrm{g} / \mathrm{mL})$ methanol. The extracts of the samples were subsequently filtered through a $0.45 \mu \mathrm{m}$ nylon membrane filter (Tianjin Jinteng Experiment Equipment China) prior to HPLC injection $(20 \mu \mathrm{L})$. The samples were prepared and analyzed in three replicates. The quantity of each analyte was subsequently obtained from the corresponding calibration curve.

\subsection{Validation of Method}

The Salix bordensis sample from Zhangye City, Gansu Province were used for the validation of the proposed method. A number of experimental parameters were studied for the methods, namely, precision, repeatability, regression linearity, stability, limit of detection (LOD), limit of quantification (LOQ), and recovery.

Linearity was determined using the square correlation coefficients of the calibration curves generated by three repeated injections of standard solutions at six concentration levels. The chromatographic conditions described above were used to analyze the Salix bordensis. The analyte peak areas obtained were plotted against the corresponding concentrations of the standard solutions, and the calibration curves were set up using the least-square method. The lowest concentration of the working solution was diluted with $80 \%$ methanol for a series of appropriate concentrations to determine the LOD and LOQ at signal-to-noise ratios of 3 and 10 for standard solutions, respectively.

Intra- and inter-day variations were investigated by analyzing known concentrations of the four analytes in six replicates in a single day and by duplicating the experiments for three successive days. The relative standard deviation (RSD) was taken as a measure of precision.

Repeatability was assessed by analyzing five independently prepared samples via the same method. Sample stability was evaluated at room temperature and analyzed at $0,2,4,8,12$, and $24 \mathrm{~h}$ within 1 day. Variations were expressed as RSD.

A recovery test was used to evaluate the accuracy of the proposed method. Accuracy was determined by adding the mixed standard solution at low (80\% of the known amounts), medium (same as the known amounts), and high (120\% of the known amounts) amounts to a Salix bordensis Turcz. sample with known concentrations. The resulting samples were then extracted and analyzed via the proposed procedure. The quantity of each analyte was subsequently obtained from the corresponding calibration curve. The recovery was calculated as follows:

Recovery $(\%)=100 \times($ Amount found - Original amount $) /$ Amount spiked.

\section{Results and Discussion}

\subsection{Optimization of Analysis Conditions}

The first step in the quantitation of the five flavonoids in Salix bordensis Turcz. (structures shown in the Table 1) is the full extraction from the materials. The preliminary tests made in this study indicated that the different extraction methods (ultrasonication, reflux, or Soxhlet) have a great influence on the content of the five flavonoids. As shown in Figure 1, the contents of the five flavonoids are higher when subjected to the ultrasonication extraction method compared with those subjected to other methods. The extraction solvent (methanol, ethanol, and ethyl acetate), extraction solvent concentration (30\% methanol, 50\% methanol and $80 \%$ methanol), solvent volume (1:8, 1:10, or 1:12 medicinal materials/solvent, $\mathrm{m} / \mathrm{v} \mathrm{g} / \mathrm{mL})$, and ultrasonication extraction time $(30,40,50$, or $60 \mathrm{~min}$ ) were designed and evaluated in this experiment by comparing the sums and contents of characteristic peaks in each chromatogram of different factors. The sample that was ultrasonically extracted for 40 min with ten times $80 \%(\mathrm{~m} / \mathrm{v} \mathrm{g} / \mathrm{mL})$ methanol resulted in the best extraction process.

\subsection{Optimization of the Chromatographic Separation by HPLC}

The Salix bordensis Turcz. is a challenging matrix because of the complexity of water-soluble compounds, such as flavonoid aglycones. Hence, the optimization of HPLC separation for quantitative analysis was difficult because of the complex chromatographic profile. The preliminary tests made in this study indicated that a longer 
column should be used in the presence of $\mathrm{f} 5$. Therefore, to optimize the resolution and run time, several mobile phases, including $\mathrm{MeOH}$-water, ACN-water, and $\mathrm{MeOH}-\mathrm{ACN}-$ water were tested on a 250-mm long C18 column. The other serious problem in the separation of flavonoids is peak tailing, which is related with the dissociation of the hydroxyl groups. The presence of acid in a mobile phase can prevent this effect by changing the $\mathrm{pH}$, thereby improving the peak symmetry of the analytes [19] [20]. As shown in Figure 2 (there are four section A, $\mathrm{a}, \mathrm{B}$ and $\mathrm{b}$ in Figure 2), the routine addition of $0.3 \%$ formic acid to the mobile phase was only sufficient to reduce tailing in real Salix bordensis extracts (shown in section A of $0.2 \%$ formic acid and section a of $0.3 \%$
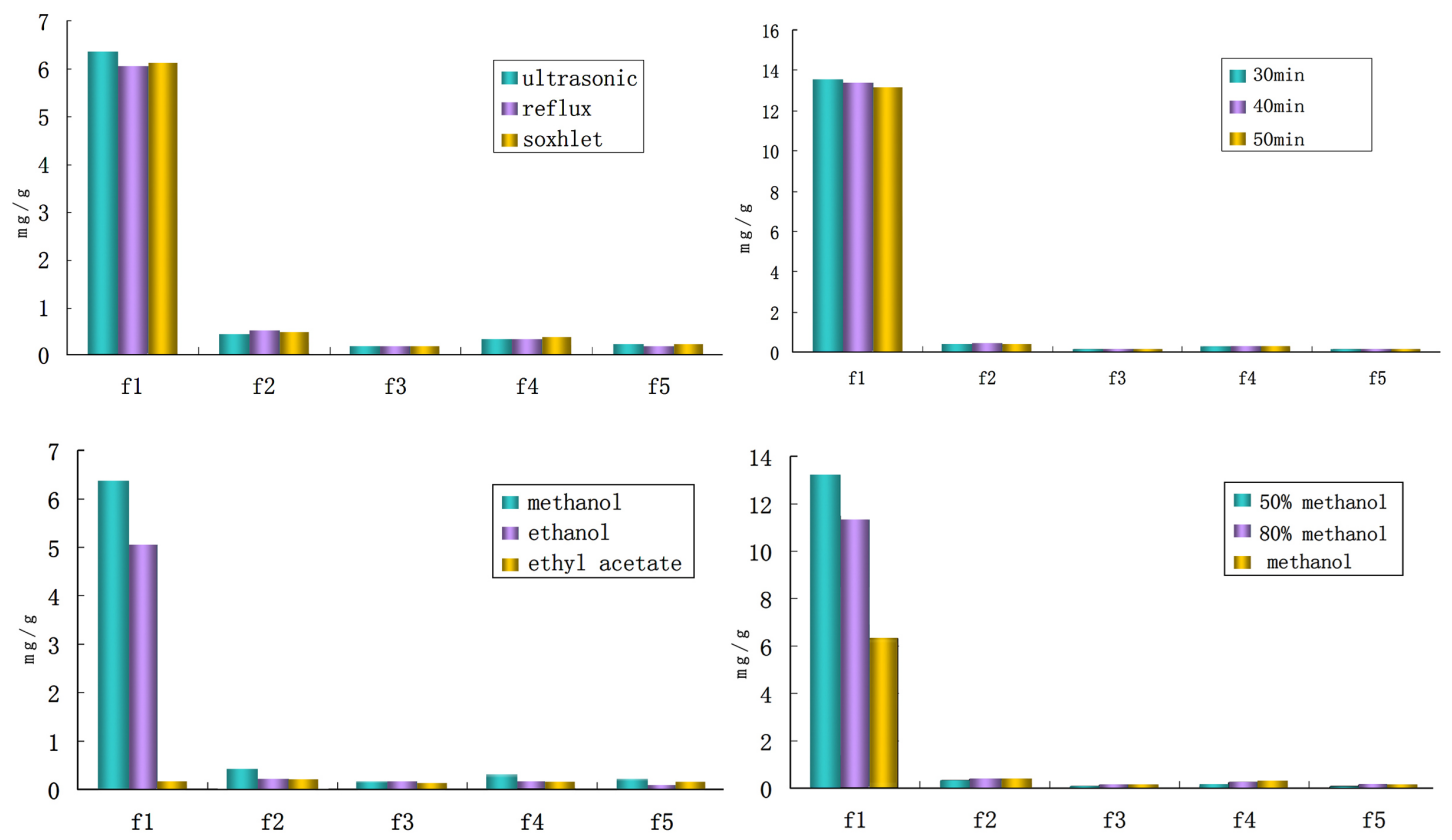

Figure 1. Effect of the different extraction method in the sample solution on the content of the five quantified compounds. All data are the mean of a triplicate analysis.
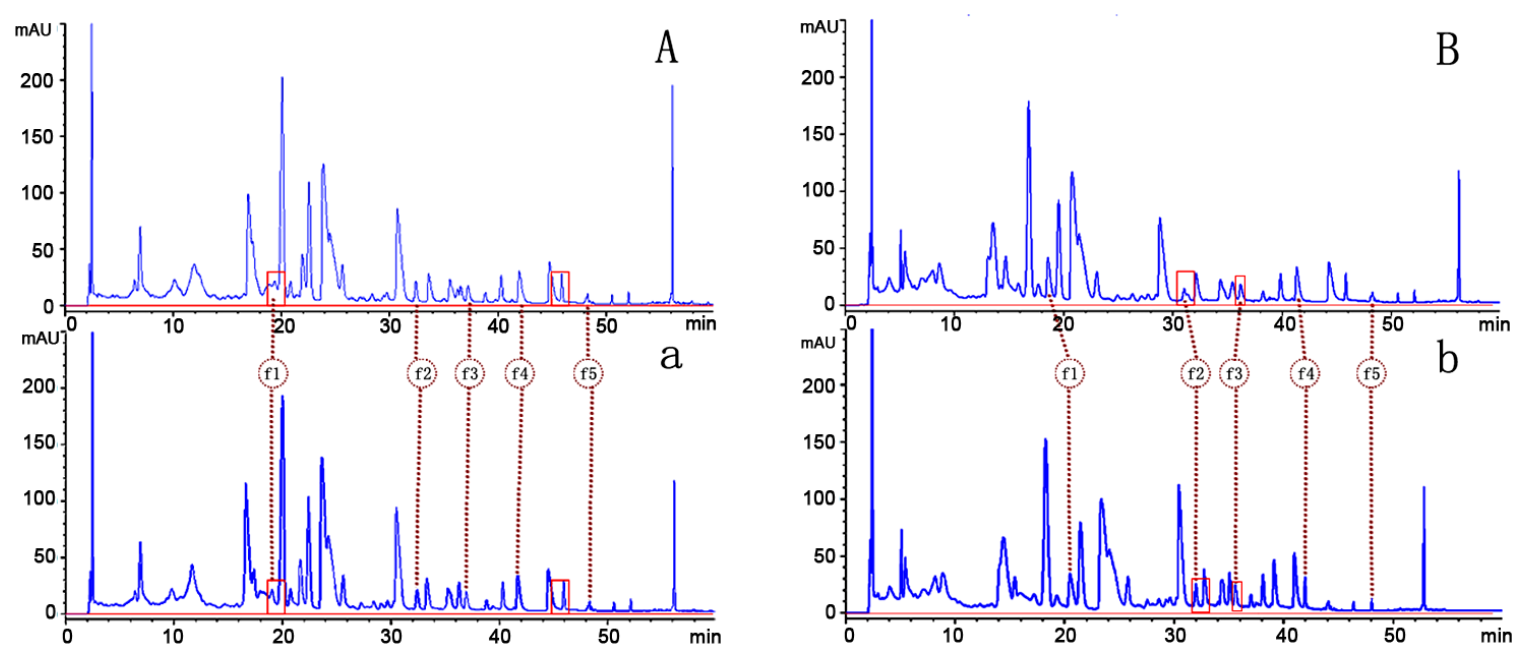

Figure 2. Chromatograms of the separation of the five quantified compounds of extracts of the Salix bordensis Turcz. (A) $0.2 \%(\mathrm{v} / \mathrm{v})$ formic acid in water as solvent $\mathrm{A}$, methanol as solvent $\mathrm{B}$, solvent gradient program as following: 0min: 28\% B; 25 min: 46\% B; 40 min: 60\% B; 53 min: 100\% B; (a) 0.3\% (v/v) formic acid in water as solvent A, methanol as solvent B, solvent gradient program the same as (A); (B) $0.3 \%(v / v)$ formic acid in water as solvent $A$, methanol as solvent B, solvent gradient program as following: 0min: 28\% B; 25 min: 46\% B; 40 min: 60\% B; 53 min: 100\% B, (b) $0.3 \%(\mathrm{v} / \mathrm{v})$ formic acid in water as solvent $\mathrm{A}$, methanol as solvent $\mathrm{B}$, solvent gradient program as following: $0 \mathrm{~min}$ : $32 \%$ B; 20 min: 40\% B; 35 min: 60\% B; 50 min: 100\% B. 
formic acid to the mobile phase of Figure 2). Different elution programmings (shown in section $B$ and section $b$ of Figure 2) with $0.3 \%$ (v/v) formic acid in water (A) and methanol (B) as the mobile phase were tested. Finally, a valid and stable HPLC was achieved through a gradient system mobile phase (shown in Table 2) at the conditions described above. The representative DAD $(360 \mathrm{~nm})$ chromatograms for the standard solutions of the five flavonoids and for the solution of inflorescences of Salix bordensis Turcz. are shown in Sections a and b of Figure 3, respectively.

\subsection{Identification of the Five Bioactive Flavonoids}

The five flavonoids in the mixture were identified by comparing their ultraviolet spectra of HPLC-DAD, retention time, and MS data with those of the corresponding reference compounds.

Diode array detection allowed the online recording of UV spectra and rapid attribution of peaks, which corresponded to the flavonoids in the chromatograms. The assignment of compounds peaks was unambiguous because these peaks exhibited characteristic UV spectra, with two absorption bands (Band I, $\lambda_{\max }$ approximately $320 \mathrm{~nm}$ to $360 \mathrm{~nm}$ and Band II, $\lambda_{\max }$ approximately $250 \mathrm{~nm}$ to $280 \mathrm{~nm}$ ). The UV spectra of the five bioactive flavonoids in the mixture were in agreement with those of their authentic reference compounds. Their UV spectra are shown in Figure 3. The peak purity of the flavonoids was determined by overlapping the UV/DAD spectra in different regions of the same peak by using the HPLC software. On the other hand, the assignment of HPLC peaks was confirmed by comparing the retention times with those of the authentic standards (Figure 3).

To confirm the above identification further and to determine a rapid and exact method that could be used to identify directly the five bioactive flavonoids in the mixture, HPLC-ESI/MS experiments were employed for online identification in the study. Positive and negative ion modes were utilized for the compounds, and the results suggested that the positive ion mode was more sensitive. The ESI source gave $[\mathrm{M}+1]^{+}$and $[\mathrm{M}+\mathrm{Na}]^{+}$ions as quasimolecular ions. Figure 4 shows that the molecular masses for peaks f1, f2, f3, f4, and f5 are 478, 302, 286, 316, and 314, respectively.

\subsection{Validation of Methodology}

The regression equation for each reference standard, along with the LOD and LOQ values, are shown in Table 3. The calibration curves show good linearity $\left(r^{2} \geq 0.9998\right)$ within the test ranges. The overall intra- and inter-day precision RSD values were higher than $1.33 \%$ and $2.44 \%$, respectively. The overall stability over $24 \mathrm{~h}$ was $4.62 \%$, and the repeatability variation was $4.62 \%$ (shown in Table 4). The developed analytical method showed excellent accuracy, and the overall recovery for all compounds was between $95.5 \%$ and $103.0 \%$ (RSD $\leq 2.34 \%$ ) (shown in Table 5). Therefore, the method is precise, accurate, and sufficiently sensitive for the simultaneous quantitative analysis of the five main bioactive flavonoids in Salix bordensis Turcz.

\subsection{Analysis of the Five Bioactive Flavonoids in Salix bordensis Turcz.}

The established HPLC-MS/MS and HPLC-DAD method was applied to the simultaneous determination of five bioactive flavonoids in Salix bordensis Turcz. The representative chromatograms of the extracts of Salix bordensis are shown in Figure 3. The symmetric factors $(0.95-1.21)$ and tailing factors $(0.93-1.13)$ showed that the investigated chromatographic peaks were sharp and symmetrical. The contents of the investigated compounds were calculated by individual calibration curves, and the mean values for three parallel determinations are summarized in Table 6. The six primary compounds were detected in the samples of Salix bordensis Turcz. Although the chemical constituents of the three samples were quite similar, several differences were observed in the contents of the five investigated flavonoids. The total amount of flavonoid ranged from $2.56 \mathrm{mg} / \mathrm{g}$ to 3.71 $\mathrm{mg} / \mathrm{g}$, which indicates that the total amount of flavonoids from Jiuquan is higher than those from other areas. Thus, the resources of Jiuquan deserve attention because of the higher content of flavonoids. Furthermore, this study showed that a number of flavonoids exist in the Salix bordensis Turcz. Thus, further investigations are needed to understand the biological activity of these resources to provide a scientific basis for exploiting this plant resource.

\section{Conclusion}

In this study, HPLC-UV/DAD and HPLC-MS/MS methods proved to be suitable and reliable for the qualitative 


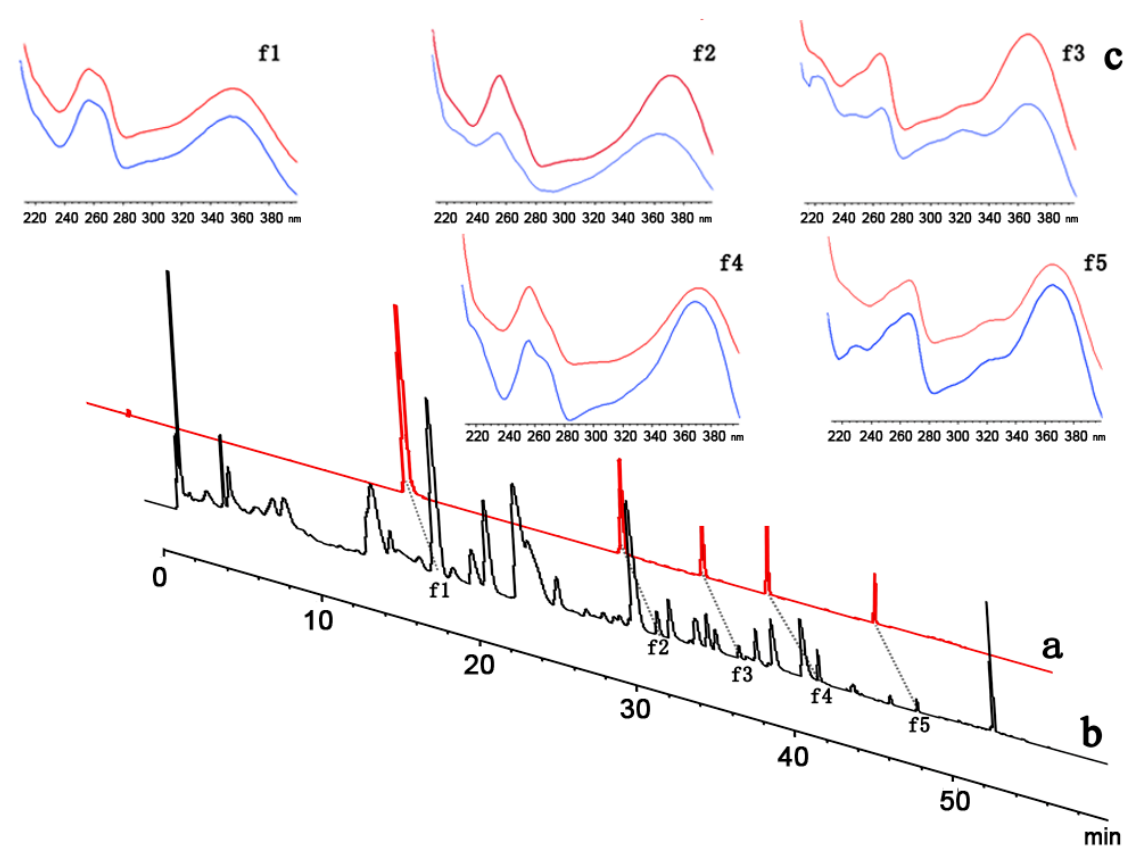

Figure 3. HPL C chromatogram recorded at $360 \mathrm{~nm}$ of mixed reference standards: Quercetin-3-O- $\beta$-D-glucoside (f1), Quercetin (f2), Kaempferol (f3), Rhamnetin (f4), Kaempferol-7,4'-dimethyl (f5) (a); and HPLC chromatogram of the 80\% methanol extracts of the Salix bordensis Turcz. (b); Ultraviolet spectrum of the five quantified compounds, the upper from the HPLC chromatograms of the $80 \%$ methanol extracts of the Salix bordensis Turcz. and the nether from the HPLC chromatograms of mixed reference standards (c).

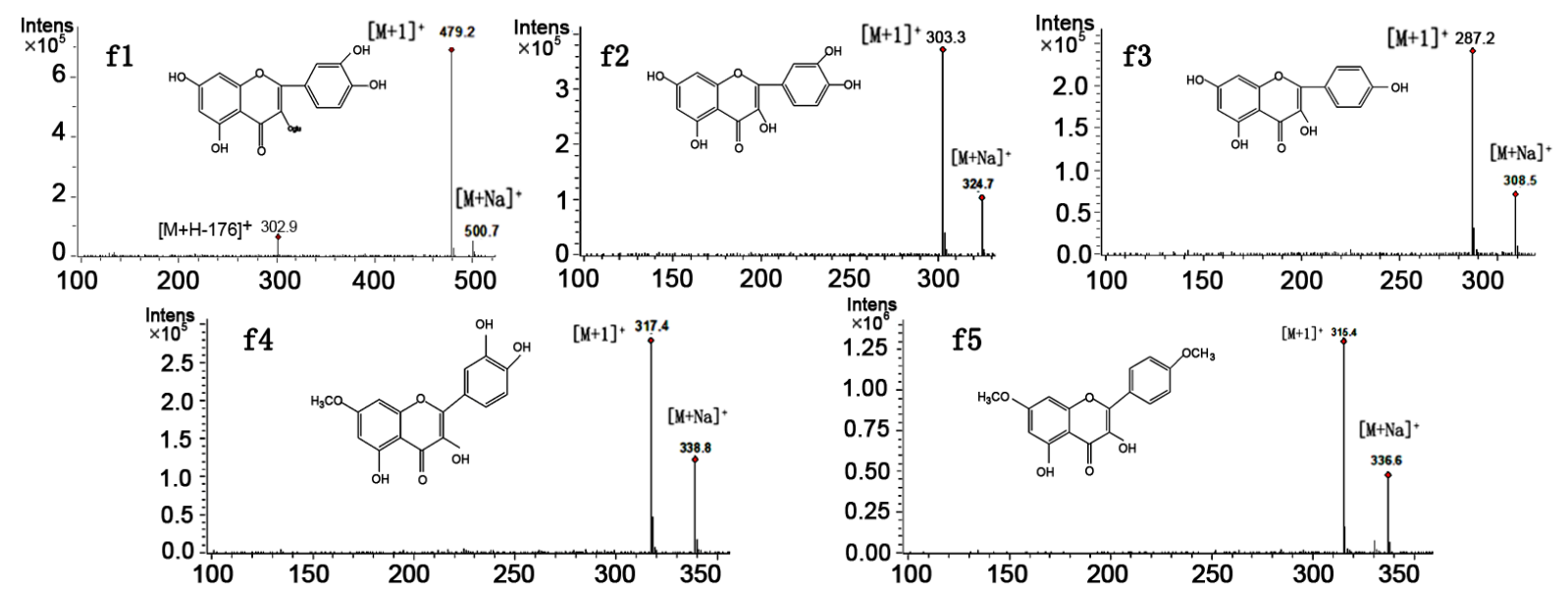

Figure 4. MS spectra of peaks f1, f2, f3, f4 and f5 of the Salix bordensis Turcz. sample on HPLC-MS/MS.

Table 3. Calibration curves of the five quantified reference standard compounds.

\begin{tabular}{ccccccc}
\hline Peak No. & Compounds & Regression Equation & Linear Range $(\mu \mathrm{g} / \mathrm{mL})$ & $\mathrm{r}^{2}$ & LOD $^{\mathrm{b}}(\mu \mathrm{g} / \mathrm{mL})$ & $\mathrm{LOQ}^{\mathrm{c}}(\mu \mathrm{g} / \mathrm{mL})$ \\
\hline 1 & $\mathrm{f} 1$ & $\mathrm{Y}=197.12 \mathrm{x}+59.629$ & $24-240$ & 0.9998 & 0.85 & 2.84 \\
2 & $\mathrm{f} 2$ & $\mathrm{Y}=83.833 \mathrm{x}-3.1994$ & $4.16-41.6$ & 0.9999 & 0.07 & 0.23 \\
3 & $\mathrm{f} 3$ & $\mathrm{Y}=43.495 \mathrm{x}-2.9818$ & $1.64-16.4$ & 0.9999 & 0.12 & 0.41 \\
4 & $\mathrm{f} 4$ & $\mathrm{Y}=42.19 \mathrm{x}-4.4204$ & $1.56-15.6$ & 0.9999 & 0.06 & 0.19 \\
5 & $\mathrm{f} 5$ & $\mathrm{Y}=22.418 \mathrm{x}-3.4394$ & $1.32-13.2$ & 0.9998 & 0.04 & 0.14 \\
6 & $\mathrm{t} 6$ & $\mathrm{Y}=73.559 \mathrm{x}-0.8748$ & $42.88-428.8$ & 0.9999 & 1.26 & 4.19 \\
\hline
\end{tabular}

${ }^{\mathrm{a} x}$, amount of substance $(\mu \mathrm{g})$; $\mathrm{y}$, peak area; ${ }^{\mathrm{b}} \mathrm{LOD}$, limit of detection $(\mathrm{S} / \mathrm{N}=3)$; ${ }^{\mathrm{C}} \mathrm{LOQ}$, limit of quantification $(\mathrm{S} / \mathrm{N}=10)$. 
Table 4. Precision, stability and repeatablity of the five quantified compounds.

\begin{tabular}{|c|c|c|c|c|c|c|c|c|c|}
\hline \multirow{3}{*}{ Peak No. } & \multirow{3}{*}{ Compounds } & \multicolumn{4}{|c|}{ Precision R.S.D. $(\%)(n=6)$} & \multirow{2}{*}{\multicolumn{2}{|c|}{ Stability $(n=6)$}} & \multirow{2}{*}{\multicolumn{2}{|c|}{ Repeatability $(\mathrm{n}=5)$}} \\
\hline & & \multicolumn{2}{|c|}{ Intra-day } & \multicolumn{2}{|c|}{ Inter-day } & & & & \\
\hline & & $\mathrm{RT}$ & Peak area & $\mathrm{RT}$ & Peak area & Mean (mg/g) & RSD (\%) & Mean (mg/g) & RSD (\%) \\
\hline 1 & $\mathrm{f} 1$ & 0.17 & 1.33 & 0.53 & 2.44 & 2.17 & 4.44 & 2.22 & 4.26 \\
\hline 2 & $\mathrm{f} 2$ & 0.07 & 1.20 & 0.28 & 2.13 & 0.17 & 4.62 & 0.18 & 3.52 \\
\hline 3 & f3 & 0.05 & 1.07 & 0.25 & 1.61 & 0.03 & 4.31 & 0.03 & 3.85 \\
\hline 4 & $\mathrm{f} 4$ & 0.02 & 1.16 & 0.21 & 1.82 & 0.07 & 4.61 & 0.08 & 2.88 \\
\hline 5 & f5 & 0.005 & 1.19 & 0.17 & 1.94 & 0.05 & 4.03 & 0.06 & 2.55 \\
\hline 6 & t6 & 0.002 & 1.12 & 0.13 & 1.76 & 3.36 & 4.40 & 3.56 & 2.35 \\
\hline
\end{tabular}

${ }^{\mathrm{a}}(\mathrm{SD} /$ mean $) \times 100$.

Table 5. Recoveries of the five quantified compounds.

\begin{tabular}{|c|c|c|c|c|c|c|}
\hline Peak no. & Compounds & Initial mean content (mg) & Added (mg) & Detected (mg) & Recovery $^{\mathrm{a}}(\%)$ & R.S.D. $^{\mathrm{b}}(\%)$ \\
\hline \multirow{3}{*}{1} & \multirow{3}{*}{ f1 } & \multirow{3}{*}{1.152} & 0.922 & 2.072 & 99.8 & \multirow{3}{*}{1.86} \\
\hline & & & 1.152 & 2.338 & 103.0 & \\
\hline & & & 1.382 & 2.531 & 99.7 & \\
\hline \multirow{3}{*}{2} & \multirow{3}{*}{ f2 } & \multirow{3}{*}{0.178} & 0.142 & 0.318 & 98.9 & \multirow{3}{*}{1.69} \\
\hline & & & 0.178 & 0.352 & 97.8 & \\
\hline & & & 0.214 & 0.394 & 101.1 & \\
\hline \multirow{3}{*}{3} & \multirow{3}{*}{ f3 } & \multirow{3}{*}{0.027} & 0.022 & 0.048 & 96.3 & \multirow{3}{*}{2.19} \\
\hline & & & 0.028 & 0.055 & 100.0 & \\
\hline & & & 0.032 & 0.058 & 96.3 & \\
\hline \multirow{3}{*}{4} & \multirow{3}{*}{$\mathrm{f} 4$} & \multirow{3}{*}{0.082} & 0.066 & 0.147 & 98.8 & \multirow{3}{*}{1.42} \\
\hline & & & 0.083 & 0.164 & 98.7 & \\
\hline & & & 0.098 & 0.181 & 101.2 & \\
\hline \multirow{3}{*}{5} & \multirow{3}{*}{ f5 } & \multirow{3}{*}{0.067} & 0.054 & 0.120 & 98.5 & \multirow{3}{*}{2.34} \\
\hline & & & 0.068 & 0.132 & 95.5 & \\
\hline & & & 0.081 & 0.148 & 100.0 & \\
\hline
\end{tabular}

a[(Mean of measured concentration-spiked concentration)/spiked concentration $] \times 100 ;{ }^{b}($ S.D. $/$ mean $) \times 100$.

Table 6. The contents (mg/g) of the five quantified compounds in Salix bordensis Turcz. from Gansu province.

\begin{tabular}{ccccccccc}
\hline \multicolumn{2}{c}{ Samples } & \multicolumn{7}{c}{ Mean $\left(\mathrm{mg} / \mathrm{g}, \mathrm{n}=3^{\mathrm{a}}\right)$} \\
\hline No. $^{\mathrm{b}}$ & Sources & $\mathrm{f} 1$ & $\mathrm{f} 2$ & $\mathrm{f} 3$ & $\mathrm{f} 4$ & $\mathrm{f} 5$ & Total $^{\mathrm{d}}$ & \\
1 & Jiuquan & $3.14^{\mathrm{c}}$ & 0.26 & 0.07 & 0.13 & 0.11 & 3.71 & 3.23 \\
2 & Wuwei & 2.76 & 0.22 & 0.05 & 0.11 & 0.09 & 0.06 & 2.56 \\
\hline
\end{tabular}

${ }^{\mathrm{a}} \mathrm{RD}$, relative deviation $(\%)=(\mid$ measured value mean/mean $) \times 100 .{ }^{\mathrm{b}}$ Salix bordensis $\mathrm{T}$. from Gansu province. ${ }^{\mathrm{c}}$ The data were presented as average of three replicates. ${ }^{\mathrm{d}}$ The sum of five compounds including quercetin-3-O- $\beta$-D-glucopyranuronide, quercetin, kaempferol, rhamnetin, kaempferol-7,4'dimethyl. 
and quantitative analyses of five bioactive flavonoids in Salix bordensis Turcz. The evaluated parameters demonstrated that the methods offer good specificity, linearity, accuracy, and precision within acceptable limits, and the LOD and LOQ confirmed the efficiency of the methodologies in quantifying low concentrations of flavonoids, thereby revealing that the comprehensive evaluation method effectively controls the quality of Salix bordensis Turcz. on a regular HPLC system. This research also showed that a number of flavonoids exist in the Salix bordensis Turcz., thereby providing a useful basis for the utilization of this plant resource.

\section{Acknowledgements}

The authors gratefully acknowledge the Fund of National Nature Science Foundation Projects (81060327), (81260616), the Fund of the Scientific and Technical Innovation Project of Gansu province (1104FKCA122, 1305TCYA043) and the Basic scientific research Fund of Colleges and Universities in Gansu province (2011-06) and Found of Key Laboratory of Pharmacology and Toxicology for Traditional Chinese Medicines of Gansu Province, Gansu College of Traditional Chinese Medicine (ZDSYS-ZZKJ-2013(A)-001) for financially supporting this research. The authors thank Prof. Chong Li for providing reference standards.

\section{References}

[1] Hughes, R.J., Croley, T.R., Metcalfe, C.D. and March, R.E. (2001) A Tandem Mass Spectrometric Study of Selected Characteristic Flavonoids. International Journal of Mass Spectrometry, 210-211, 371-385. http://dx.doi.org/10.1016/S1387-3806(01)00451-1

[2] March, R.E., Miao, X.S., Metcalfe, C.D., Stobiecki, M. and Marczak, L. (2004) A Fragmentation Study of an Isoflavone Glycoside, Genistein-7-O-Glucoside, Using Electrospray Quadrupole Time-of-Flight Mass Spectrometry at High Mass Resolution. International Journal of Mass Spectrometry, 232, 171-183. http://dx.doi.org/10.1016/j.ijms.2004.01.001

[3] Hämäläinen, M., Nieminen, R., Vuorela, P., Heinonen, M. and Moilanen, E. (2007) Anti-Inflammatory Effects of Flavonoids: Genistein, Kaempferol, Quercetin, and Daidzein Inhibit STAT-1 and NF-кB Activations, Whereas Flavone, Isorhamnetin, Naringenin, and Pelargonidin Inhibit Only NF-кB Activation along with Their Inhibitory Effect on iNOS Expression and NO Production in Activated Macrophages. Mediators of Inflammation, 2007, 45673-45683.

[4] Labbé, D., Provençal, M., Lamy, S., Boivin, D., Gingras, D. and Béliveau, R. (2009) The Flavonols Quercetin, Kaempferol, and Myricetin Inhibit Hepatocyte Growth Factor-Induced Medulloblastoma Cell Migration. Journal of Nutrition, 139, 646-652. http://dx.doi.org/10.3945/jn.108.102616

[5] Ackland, M.L., van de Waarsenburg, S. and Jones, R. (2005) Synergistic Antiproliferative Action of the Flavonols Quercetin and Kaempferol in Cultured Human Cancer Cell Lines. In Vivo, 19, 69-76.

[6] Schmidt, S., Zietz, M., Schreiner, M., Rohn, S., Kroh, L.W. and Krumbein, A. (2010) Identification of Complex, Naturally Occurring Flavonoid Glycosides in Kale (Brassica oleracea var. sabellica) by High-Performance Liquid Chromatography Diode-Array Detection/Electrospray Ionization Multi-Stage Mass Spectrometry. Rapid Communications in Mass Spectrometry, 24, 2009-2022. http://dx.doi.org/10.1002/rcm.4605

[7] Boots, A.W., Haenen, G.R. and Bast, A. (2008) Health Effects of Quercetin: From Antioxidant to Nutraceutical. European Journal of Pharmacology, 585, 325-337. http://dx.doi.org/10.1016/j.ejphar.2008.03.008

[8] Formica, J.V. and Regelson, W. (1995) Review of the Biology of Quercetin and Related Bioflavonoids. Food and Chemical Toxicology, 33, 1061-1080. http://dx.doi.org/10.1016/0278-6915(95)00077-1

[9] Kook, D., Wolf, A.H., Yu, A.L., Neubauer, A.S., Priqlinger, S.G., Kmpik, A. and Welge-Lüssen, U.C. (2008) The Protective Effects of Quercetin against Oxidative Stress in the Human RPE in Vitro. Investigative Ophthalmology \& Visual Science, 49, 1712-1720. http://dx.doi.org/10.1167/iovs.07-0477

[10] Duarte, J., Pérez-Vizcaíno, F., Zarzuelo, A., Jiménez, J. and Tamargo, J. (1993) Vasodilator Effects of Quercetin in Isolated Rat Vascular Smooth Muscle. European Journal of Pharmacology, 239, 1-7. http://dx.doi.org/10.1016/0014-2999(93)90968-N

[11] Nishida, S. and Satoh, H. (2009) Possible Involvement of $\mathrm{Ca}^{2+}$ Activated $\mathrm{K}^{+}$Channels, SK Channel, in the QuercetinInduced Vasodilatation. Korean Journal of Physiology and Pharmacology, 13, 361-365. http://dx.doi.org/10.4196/kjpp.2009.13.5.361

[12] Liren, S. (2009) Chinese Materia Medica. Shanghai Science and Technology Press, Shanghai, 406.

[13] Dong, J.F., Li, C.H., Liu, G.H., Zhou, S.Q. and Su, Y.L.T. (2009) Analysis of Drought Resistance by Leaf Anatomical Structure of Six Species of Sandy Willows. Journal of Desert Research, 29, 480-494.

[14] Guo, J.Y., Yu, W., Hu, X.L. and Wang, L.B. (2005) Ecological Research on Shrub Vegetation in Hunshandake Sandy 
Land. Inner Mongolia Forestry Science \& Technology, 4, 4-8.

[15] Zhao, H.L., Guo, Y.R., Zhou, R.L. and Zhao, X.Y. (2009) Effects of Vegetation Cover on Physical and Chemical Properties of Bio-Crust and Under-Layer Soil in Horqin Sand Land. Ying Yong Sheng Tai Xue Bao, 20, 1657-1663.

[16] Liu, M.Z., Jiang, G.M., Li, Y.G., Gao, L.M., Niu, S.L., Cui, H.X. and Ding, L. (2003) Gas Exchange, Photochemical Efficiency, and Leaf Water Potential in Three Salix Species. Photosynthetica, 41, 393-398. http://dx.doi.org/10.1023/B:PHOT.0000015463.04706.f3

[17] Luo, J.J. (2013) Chemical Consitituents of Salix bordensis. Lanzhou University, Lanzhou, 43-62.

[18] Yang, H.P. (2003) Studies on Constituents of Fruit Body of Polyporus umbellatus and Salix bordensis. Northwest Normal University, Lanzhou, 53-60.

[19] Hasler, A., Sticher, O. and Meier, B. (1990) High-Performance Liquid Chromatographic Determination of Five Widespread Flavonoid Aglycones. Journal of Chromatography A, 508, 236-240. http://dx.doi.org/10.1016/S0021-9673(00)91262-7

[20] Zu, Y., Li, C., Fu, Y. and Zhao, C. (2006) Simultaneous Determination of Catechin, Rutin, Quercetin Kaempferol and Isorhamnetin in the Extract of Sea Buckthorn (Hippophae rhamnoides L.) Leaves by RP-HPLC with DAD. Journal of Pharmaceutical and Biomedical Analysis, 41, 714-719. http://dx.doi.org/10.1016/j.jpba.2005.04.052 
Scientific Research Publishing (SCIRP) is one of the largest Open Access journal publishers. It is currently publishing more than 200 open access, online, peer-reviewed journals covering a wide range of academic disciplines. SCIRP serves the worldwide academic communities and contributes to the progress and application of science with its publication.

Other selected journals from SCIRP are listed as below. Submit your manuscript to us via either submit@scirp.org or Online Submission Portal.
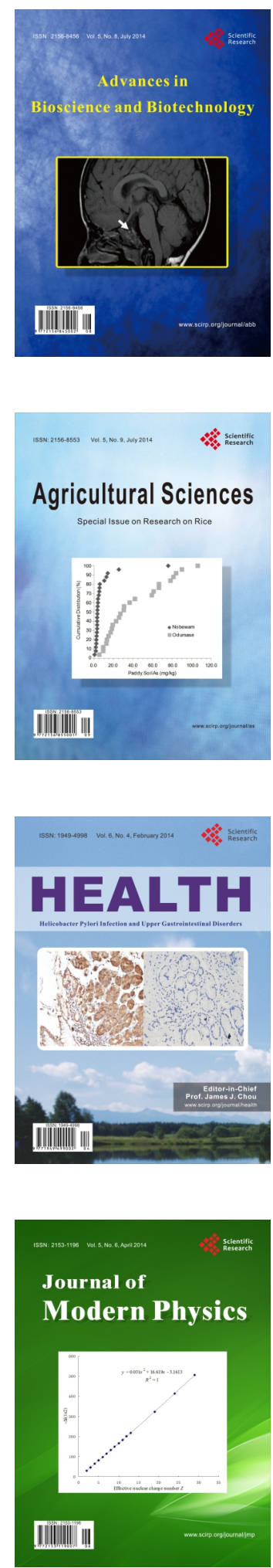
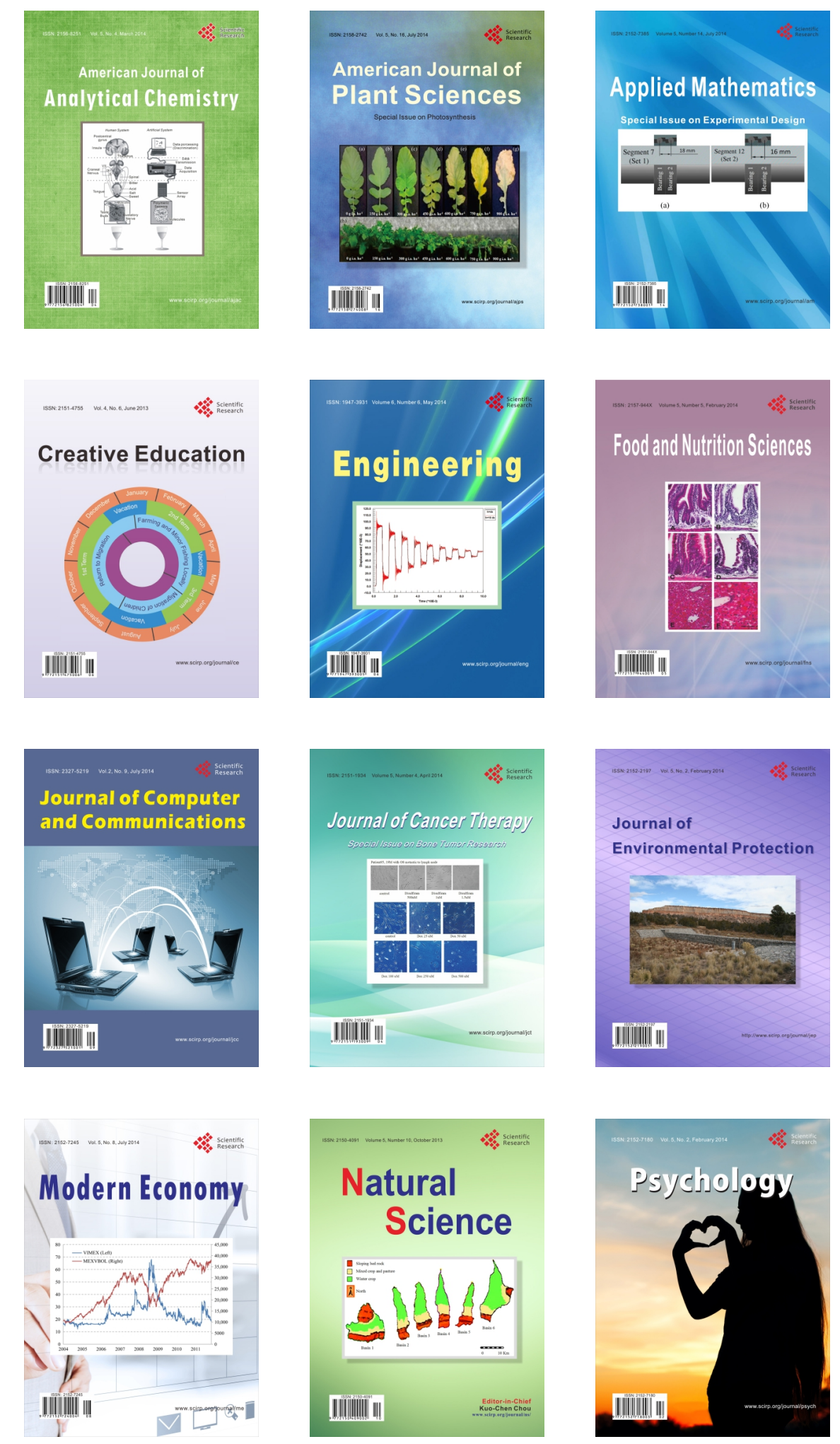